\title{
An effective lower bound for the height of algebraic numbers
}

\author{
by
}

Paul Voutier (London)

1. Introduction. Let $\alpha$ be a non-zero algebraic number of degree $d$ with

$$
f(X)=a_{d} \prod_{i=1}^{d}\left(X-\alpha_{i}\right)
$$

as its minimal polynomial over $\mathbb{Z}$ and $a_{d}$ positive. We shall define the Mahler measure of $\alpha, M(\alpha)$, by

$$
M(\alpha)=a_{d} \prod_{i=1}^{d} \max \left(1,\left|\alpha_{i}\right|\right)
$$

and the absolute logarithmic height of $\alpha, h(\alpha)$, via the relationship

$$
h(\alpha)=\frac{\log M(\alpha)}{d} .
$$

In 1933, D. H. Lehmer [9] asked whether it is true that for every positive $\varepsilon$ there exists an algebraic number $\alpha$ for which $1<M(\alpha)<1+\varepsilon$. This question has since been reformulated as whether there exists a positive absolute constant $c_{0}$ such that $M(\alpha)>1+c_{0}$, if $\alpha$ is not a root of unity; in this form the question is known as Lehmer's problem. The first progress was due to Schinzel and Zassenhaus [16], who proved in 1965 that $\alpha>1+4^{-s-2}$ where $2 s$ is the number of complex conjugates of $\alpha$. This implies $M(\alpha)>1+c_{1} / 2^{d}$ for a positive absolute constant $c_{1}$. In 1971, Blanksby and Montgomery [2] used Fourier analysis to make a considerable refinement upon this first result. They proved that $M(\alpha)>1+1 /\left(52 d^{2} \log (6 d)\right)$. In 1978, C. L. Stewart [18] introduced a method from transcendental number theory to prove that $M(\alpha)>1+1 /\left(10^{4} d^{2} \log d\right)$. While this result is a little weaker than the one due to Blanksby and Montgomery, the method used has since become quite important as it has produced the best results yet known, results which are significantly better than those previously known and bring us quite close to the conjectured lower bound. 
Dobrowolski [6] was able to use Stewart's method to show that for each $\varepsilon>0$, there exists a positive integer $d(\varepsilon)$ such that for all $d \geq d(\varepsilon)$,

$$
M(\alpha)>1+\frac{1-\varepsilon}{d}\left(\frac{\log \log d}{\log d}\right)^{3} .
$$

Moreover, his proof can be made effective. Dobrowolski claims that, for all $d \geq 3$,

$$
M(\alpha)>1+\frac{1}{1200 d}\left(\frac{\log \log d}{\log d}\right)^{3} .
$$

Dobrowolski achieves his results by constructing a polynomial, $F(X) \in$ $\mathbb{Z}[X]$, of small height which is divisible by $f(X)^{T}$, for a certain integer $T$ which depends on $d$, and considering the norm of $F\left(\alpha^{p}\right)$ at certain primes $p$. The big improvement of his lower bound over previous ones arises by a clever use of Fermat's little theorem to replace the trivial lower bound $1 \leq$ $\left|\mathcal{N}_{\mathbb{Q}(\alpha) / \mathbb{Q}}\left(F\left(\alpha^{p}\right)\right)\right|$ by $p^{d T} \leq\left|\mathcal{N}_{\mathbb{Q}(\alpha) / \mathbb{Q}}\left(F\left(\alpha^{p}\right)\right)\right|$.

In 1981, Cantor and Straus [5] showed that instead of using the auxiliary function $F(X)$, one could develop a version of Dobrowolski's proof by considering the determinant of a certain matrix. Fermat's little theorem still permits a non-trivial lower bound for the absolute value of this determinant while Hadamard's inequality gives an upper bound which involves $M(\alpha)$. Comparing these bounds, as in Dobrowolski's proof, yields the desired lower bound upon judicious choice of certain parameters. Their proof had the advantages of being simpler than Dobrowolski's as well as yielding an improved result: they were able to replace the constant $1-\varepsilon$ by $2-\varepsilon$. Louboutin [10], in 1983, used a more refined selection of parameters to improve this constant to $9 / 4-\varepsilon$. In 1988, Meyer [12] showed that one can also obtain this result from Dobrowolski's method using an auxiliary function.

In addition to these results there is also a very important result due to Smyth [17]. In 1971, he proved that if $\alpha^{-1}$ is not a conjugate of $\alpha$ then $M(\alpha) \geq \alpha_{1}=1.32471 \ldots$ This number, $\alpha_{1}$, is the real root of the polynomial $X^{3}-X-1$ and also the smallest Pisot number. One consequence of his result is the positive solution of Lehmer's problem when $d$ is odd with $c_{0}=0.32471 \ldots$, since reciprocal polynomials (those with $\alpha^{-1}$ as a root whenever $\alpha$ is a root) of odd degree have -1 as a root and are hence reducible. Therefore, it is now only necessary to consider even values of $d$.

Finally, let us mention some computational work done on this problem. Lehmer performed considerable computations in the early thirties. The smallest value of $M(\alpha)$ he was able to find arose from the example $\alpha=$ $M(\alpha)=1.1762808 \ldots$ which is a root of the polynomial

$$
X^{10}+X^{9}-X^{7}-X^{6}-X^{5}-X^{4}-X^{3}+X+1 .
$$


This value of $M(\alpha)$ is still the smallest known - indeed, it is widely believed that this is the minimum value of $M(\alpha)$. Interestingly, this $\alpha$ is also the smallest known Salem number.

Boyd $[3,4]$ has conducted extensive calculations on this problem and determined all $\alpha$ of degree at most 20 with $M(\alpha) \leq 1.3$ without finding smaller values. Thus, we need only consider even values of $d \geq 22$.

We mentioned before that Dobrowolski's proof can be made effective. So can those of Cantor and Straus and Louboutin. In this article, we produce such an effective result using the method of Cantor and Straus. Using their proof has the advantage of simplicity over the other two methods. Moreover, although it yields a result which is asymptotically weaker than Louboutin's lower bound, for small $d$ the methods give rise to the same choice of parameters and so we do not lose anything by this choice. Let us now state our result.

TheOREM. Suppose $\alpha$ is a non-zero algebraic number of degree $d$ which is not a root of unity. If $d \geq 2$ then

$$
d h(\alpha)=\log M(\alpha)>\frac{1}{4}\left(\frac{\log \log d}{\log d}\right)^{3} .
$$

There is a conjecture related to Lehmer's problem due to Schinzel and Zassenhaus. They ask whether there exists a positive absolute constant $c_{1}$ such that the maximum of the absolute values of the conjugates of $\alpha$, denoted by $\mid \alpha$, always satisfies $|\alpha| \geq 1+c_{2} / d$ when $\alpha$ is a non-zero algebraic integer which is not a root of unity. Notice that $1+\log M(\alpha)<M(\alpha) \leq$ $|\alpha|^{d}$ so $1+(\log M(\alpha)) / d<\mid \alpha$. Therefore, Smyth's result implies that this conjecture is true when $\alpha^{-1}$ is not an algebraic conjugate of $\alpha$. When $\alpha^{-1}$ and $\alpha$ are algebraic conjugates, we can replace the inequality above by $1+\log M(\alpha)<|\alpha|^{d / 2}$ for such $\alpha$, and so our theorem yields the following corollary.

Corollary 1. Let $\alpha$ be a non-zero algebraic number of degree $d$ which is not a root of unity. Then

$$
|\alpha|>1+\frac{1}{2 d}\left(\frac{\log \log d}{\log d}\right)^{3} .
$$

The best known result on this problem is that

$$
|\alpha|>1+\left(\frac{64}{\pi^{2}}-\varepsilon\right) \frac{1}{d}\left(\frac{\log \log d}{\log d}\right)^{3},
$$

for $d \geq d(\varepsilon)$. This result was proven in 1993 by Dubickas [7].

For $2 \leq d \leq 2300$, there is a better result than Corollary 1 which is due to Matveev [11]: for $d \geq 1$,

$$
\mid \alpha>\exp \left(\frac{3 \log (d / 2)}{d^{2}}\right) \text {. }
$$


In applications, it will often be more convenient to use a simpler form of these results. Therefore, we record the following corollary which follows from the theorem, the work of Boyd [3, 4] and Smyth [17] and the inequality $1+\log M(\alpha)<|\alpha|^{d / 2}$ when $\alpha$ and $\alpha^{-1}$ are algebraic conjugates along with the fact that, as shall be demonstrated in the course of the proof of the theorem, we may replace $1 / 4$ by 0.56 for $22 \leq d \leq 190$. Let us note that, at the expense of more effort and further complications, we could prove such a result for $d>190$ too.

Corollary 2. Suppose $\alpha$ is a non-zero algebraic number of degree $d$ which is not a root of unity. If $d \geq 2$ then

and

$$
d h(\alpha)=\log M(\alpha)>\frac{2}{(\log (3 d))^{3}}
$$

$$
|\alpha|>1+\frac{4}{d(\log (3 d))^{3}} .
$$

2. Preliminary lemmas. Let us begin with some lemmas.

LEMMA 1. If $K$ is a positive integer then

$$
\prod_{k=0}^{K-1}(k !) \geq \exp \left(\frac{K^{2} \log K}{2}-\frac{3 K^{2}}{4}\right) .
$$

Pro of. For $K=1$, the left-hand side is 1 , whereas the right-hand side is $0.47 \ldots$ So we may assume that $K \geq 2$.

In the course of proving their Lemma 8, Laurent, Mignotte and Nesterenko [8] showed that

$$
\begin{aligned}
\prod_{k=0}^{K-1}(k !) \geq \exp \left(\frac{\left(K^{2}-K\right) \log (K-1)}{2}-\frac{3\left(K^{2}-K\right)}{4}\right. & \\
& \left.+\frac{K \log (2 \pi(K-1) / \sqrt{e})}{2}-\frac{\log K}{12}\right) .
\end{aligned}
$$

By means of the relation $\log (K-1)=\log K+\log (1-1 / K)$ and the series expansion for $\log (1-x)$, we can replace the two terms of the form $\log (K-1)$ by $\log K-7 /(5 K)$ for $K \geq 2$ to obtain

$$
\prod_{k=0}^{K-1}(k !) \geq \exp \left(\frac{K^{2} \log K}{2}-\frac{3 K^{2}}{4}+K \log \left(\sqrt{2 \pi} e^{-1 / 5}\right)-\frac{\log K}{12}\right) .
$$

The lemma now follows.

LemMA 2. Let $p_{1}=2, p_{2}=3, \ldots$ denote the prime numbers in increasing order. 
(i) For $S \geq 13$,

$$
\theta\left(p_{S}\right)=\sum_{i=1}^{S} \log p_{i} \geq S \log S
$$

(ii) For $i \geq 20$,

$$
p_{i}<i(\log i+\log \log i-1 / 2)
$$

(iii) For $S \geq 9$,

$$
\sum_{i=1}^{S} p_{i} \leq 0.564 S^{2} \log S .
$$

Pro of. (i) This is Théorème 4 of [14].

(ii) This is equation (3.11) in the statement of Theorem 3 of [15]. We will use it to prove part (iii).

(iii) One can easily check that this is true for $9 \leq S \leq 19$. In the course of this calculation we find that $p_{1}+\ldots+p_{19}=568$, a fact that we shall now use to prove the inequality in general.

For $S \geq 20$, we have

$$
\begin{aligned}
\sum_{i=1}^{S} p_{i} & <568+\sum_{i=20}^{S}(i \log i+i \log \log i-i / 2) \\
& \leq 568+\int_{i=20}^{S}(i \log i+i \log \log i-i / 2) d i+S \log S+S \log \log S-S / 2 .
\end{aligned}
$$

Knowing that

$$
\int(x \log x+x \log \log x-x / 2) d x=\frac{x^{2}}{2}(\log x+\log \log x-1)-\operatorname{li}\left(x^{2}\right) / 2,
$$

that $\operatorname{li}\left(x^{2}\right)>x^{2} /(2 \log x)$ and that $\operatorname{li}(400)=85.417 \ldots$, we find that

$$
\begin{aligned}
\sum_{i=1}^{S} p_{i}< & \frac{S^{2}}{2}\left(\log S+\log \log S-1-\frac{1}{2 \log S}\right) \\
& +S \log S+S \log \log S-S / 2-7.8 .
\end{aligned}
$$

To prove the desired inequality we want to consider

$$
f(S)=\frac{\log \log S-1-1 /(2 \log S)}{\log S}+\frac{2(S \log S+S \log \log S-S / 2-7.8)}{S^{2} \log S} .
$$

Taking the derivative of $f(S)$, we find that its maximum for $S \geq 20$, which occurs at $S=2803.26 \ldots$, is less than 0.128 . This implies our result.

We now want to give a technical lemma for later use. 
Lemma 3. Suppose $d>10000,1.2 \leq k_{1} \leq 1.6$ and $1.2 \leq s_{1}$. Then

$$
\begin{aligned}
\left(k_{1}^{2}+2 s_{1}\right) \frac{\log \log \log d}{\log \log d}+\frac{\left(0.89+\log k_{1}\right) k_{1}^{2}+2.39 s_{1}}{\log \log d} & -\left(k_{1}^{2}+2 s_{1}\right) \\
> & -\frac{2 k_{1} s_{1} \log s_{1} \log d}{(\log \log d)^{2}} .
\end{aligned}
$$

Proof. Notice that $0.89+\log 1.2>1.07$ and put

$$
\begin{aligned}
& f_{1}(d)=1-\frac{\log \log \log d}{\log \log d}-\frac{1.07}{\log \log d}, \\
& f_{2}(d)=2-\frac{2 \log \log \log d}{\log \log d}-\frac{2.39}{\log \log d} .
\end{aligned}
$$

We want to show that

$$
g\left(d, k_{1}, s_{1}\right)=\frac{2 k_{1} s_{1} \log s_{1} \log d}{(\log \log d)^{2}}-f_{1}(d) k_{1}^{2}-f_{2}(d) s_{1}
$$

is positive for $d>10000,1.2 \leq k_{1} \leq 1.6$ and $1.2 \leq s_{1}$.

We start by noting that

$$
f_{1}^{\prime}(d)=\frac{7+100 \log \log \log d}{100 d \log d(\log \log d)^{2}} \quad \text { and } \quad f_{2}^{\prime}(d)=\frac{39+200 \log \log \log d}{100 d \log d(\log \log d)^{2}},
$$

so $f_{1}$ and $f_{2}$ are increasing functions for $d \geq \exp \exp 1=15.15 \ldots$ We shall also need to know that $(\log d) /(\log \log d)^{2}$ is increasing for $d \geq \exp \exp 2=$ $1618.17 \ldots$

We will prove that $g\left(d, k_{1}, s_{1}\right)$ is positive in the desired domain by considering six different ranges of $d$.

If $10000<d \leq 10^{8}$ then $(\log d) /(\log \log d)^{2}>1.865, f_{1}(d)<0.27$ and $f_{2}(d)<0.45$ so $g\left(d, k_{1}, s_{1}\right)>g_{1}\left(k_{1}, s_{1}\right)=3.73 k_{1} s_{1} \log s_{1}-0.27 k_{1}^{2}-0.45 s_{1}$ for these $d, k_{1}>0$ and $s_{1}>1$. Notice that $g_{1}(1.2,1.2)>0.05$ and that $g_{1}\left(k_{1}, 1.2\right)$ is a quadratic polynomial which is positive between its two roots at $0.978 \ldots$ and $2.044 \ldots$ These two facts combined with the fact that $\left(\partial / \partial s_{1}\right) g_{1}\left(k_{1}, s_{1}\right)=3.73 k_{1} \log s_{1}+3.73 k_{1}-0.45$ is positive for our range of $k_{1}$ and $s_{1}$ proves that $g_{1}$, and hence $g$, is positive for these values of $d$.

In the next four intervals we proceed similarly.

For $10^{8}<d \leq 10^{12}$, we use $g_{1}=4.34 k_{1} s_{1} \log s_{1}-0.32 k_{1}^{2}-0.56 s_{1}$ and find that $g_{1}(1.2,1.2)>0.0066$, the roots of $g_{1}\left(k_{1}, 1.2\right)$ are $1.165 \ldots$ and $1.801 \ldots$ and $\left(\partial / \partial s_{1}\right) g_{1}\left(k_{1}, s_{1}\right)$ is positive for our range of $k_{1}$ and $s_{1}$ to prove the lemma.

For $10^{12}<d \leq 10^{18}$, we use $g_{1}=5.01 k_{1} s_{1} \log s_{1}-0.36 k_{1}^{2}-0.66 s_{1}$; $g_{1}(1.2,1.2)>0.0049$, the roots of $g_{1}\left(k_{1}, 1.2\right)$ are $1.179 \ldots$ and $1.865 \ldots$ and again $\left(\partial / \partial s_{1}\right) g_{1}\left(k_{1}, s_{1}\right)$ is positive for our range of $k_{1}$ and $s_{1}$, which as before establishes the lemma for this range of $d$. 
For $10^{18}<d \leq 10^{30}$, we use $g_{1}=5.97 k_{1} s_{1} \log s_{1}-0.41 k_{1}^{2}-0.76 s_{1}$. The roots of $g_{1}\left(k_{1}, 1.2\right)$ are $1.033 \ldots$ and $2.152 \ldots, g_{1}(1.2,1.2)>0.064$ and $\left(\partial / \partial s_{1}\right) g_{1}\left(k_{1}, s_{1}\right)$ is positive for our range of $k_{1}$ and $s_{1}$. This proves the lemma for this range of $d$.

For $10^{30}<d \leq 10^{100}$, we use $g_{1}=7.7 k_{1} s_{1} \log s_{1}-0.5 k_{1}^{2}-0.94 s_{1}$ and find that $g_{1}(1.2,1.2)>0.17$, the roots of $g_{1}\left(k_{1}, 1.2\right)$ are $0.921 \ldots$ and $2.447 \ldots$ and again $\left(\partial / \partial s_{1}\right) g_{1}\left(k_{1}, s_{1}\right)$ is positive for our range of $k_{1}$ and $s_{1}$ to prove the lemma.

For $d>10^{100}$, we have $f_{1}(d)<1, f_{2}(d)<2$ and $(\log d) /(\log \log d)^{2}>$ 7.78 for such $d$. Therefore, $g\left(k_{1}, s_{1}, d\right)>h\left(k_{1}, s_{1}\right)=15.56 k_{1} s_{1} \log s_{1}-k_{1}^{2}-$ $2 s_{1}$ for $k_{1}>0, s_{1}>1$ and our range of $d$. As in the previous cases, we use the facts that $h(1.2,1.2)=0.245 \ldots$, the partial derivative of $h$ with respect to $s_{1}$ is positive for $k_{1}>1$ and $s_{1}>1$, and the roots of $h\left(k_{1}, 1.2\right)$ are $0.996 \ldots$ and $2.407 \ldots$ to prove the lemma in this remaining case.

To allow us to get a good constant in the theorem, we shall make use of the following result bounding the absolute value of the discriminant of a number field in terms of its degree.

Lemma 4. Let $\mathbb{K}$ be an algebraic number field of degree $d$ with $D_{\mathbb{K}}$ as its discriminant. Then

$$
\log \left|D_{\mathbb{K}}\right|>3.108 d-8.6 d^{1 / 3} .
$$

Proof. This is a result of Odlyzko, see equation (22) of [13].

The last lemma we give before introducing the ideas of Cantor and Straus will help us to prove that a certain determinant is not zero.

Lemma 5. Suppose $\alpha$ is a non-zero algebraic integer of degree $d$ with $\alpha=\alpha_{1}, \ldots, \alpha_{d}$ as its conjugates. If there exist positive rational numbers $r$ and $s$ such that $\alpha_{i}^{r}=\alpha_{j}^{s}$ then either $r=s$ or $\alpha$ is a root of unity.

Proof. This is Lemma 2(i) of [6].

We now come to the work of Cantor and Straus.

Let $v_{0}(\beta)=\left(1, \beta, \beta^{2}, \ldots, \beta^{n-1}\right)^{t}$ and

$$
\begin{aligned}
v_{i}(\beta) & =\frac{1}{i !} \cdot \frac{d^{i}}{d \beta^{i}} v_{0}(\beta) \\
& =\left(\left(\begin{array}{c}
0 \\
i
\end{array}\right) \beta^{-i},\left(\begin{array}{c}
1 \\
i
\end{array}\right) \beta^{1-i}, \ldots,\left(\begin{array}{c}
n-1 \\
i
\end{array}\right) \beta^{n-1-i}\right)^{t} \quad \text { for } i \geq 1,
\end{aligned}
$$

where we set $\left(\begin{array}{c}h \\ i\end{array}\right)=0$ if $h<i$ and $h \in \mathbb{Z}$.

Suppose that

$$
\boldsymbol{\beta}=\left(\beta_{1}, \ldots, \beta_{m}\right) \in \mathbb{C}^{m} \quad \text { and } \quad \mathbf{r}=\left(r_{1}, \ldots, r_{m}\right) \in \mathbb{Z}_{>0}^{m} .
$$


We put $n=\sum_{j=1}^{m} r_{j}$ and define the confluent Vandermonde determinant $V(\boldsymbol{\beta}, \mathbf{r})$ to be the determinant of the $n \times n$ matrix whose columns are the vectors $v_{i}\left(\beta_{j}\right)$ where $0 \leq i \leq r_{j}-1$ and $1 \leq j \leq m$.

This $n \times n$ matrix bears some relation to the matrix associated with the system of linear equations from which Dobrowolski [6] constructs his auxiliary function. In addition, a resemblance which this matrix bears to the Vandermonde matrix yields a particularly elegant formula for its determinant.

Lemma 6. Suppose that $\boldsymbol{\beta}$ and $\mathbf{r}$ are as above. Then

$$
V(\boldsymbol{\beta}, \mathbf{r})= \pm \prod_{1 \leq i<j \leq m}\left(\beta_{i}-\beta_{j}\right)^{r_{i} r_{j}}
$$

Proof. This is Lemma 1 of [5].

Let us now see how to use this determinant to prove our theorem.

Let $k$ and $s$ be two positive integers. Put $p_{0}=1$ and let $p_{1}, \ldots, p_{s}$ be the first $s$ prime numbers. We define

$\boldsymbol{\beta}=\left(\alpha_{1}^{p_{0}}, \ldots, \alpha_{d}^{p_{0}}, \alpha_{1}^{p_{1}}, \ldots, \alpha_{d}^{p_{1}}, \ldots, \alpha_{1}^{p_{s}}, \ldots, \alpha_{d}^{p_{s}}\right), \quad \mathbf{r}=(k, \ldots, k, 1, \ldots, 1)$, where the first $d$ components of $\mathbf{r}$ are $k$ 's and the last $s d$ components are 1 's. Notice that $m=(s+1) d$ and $n=d(k+s)$.

From the previous three lemmas, we shall determine a lower bound for $|V(\boldsymbol{\beta}, \mathbf{r})|$.

Lemma 7. Suppose that $\alpha$ is a non-zero algebraic integer of degree $d \geq 2$ over $\mathbb{Q}$ which is not a root of unity and $\mathbb{Q}\left(\alpha^{p}\right)=\mathbb{Q}(\alpha)$ for all primes $p$. Moreover, suppose that $\boldsymbol{\beta}$ and $\mathbf{r}$ are as defined above. Then

$$
|V(\boldsymbol{\beta}, \mathbf{r})|^{2} \geq\left(p_{1} \ldots p_{s}\right)^{2 d k} \exp \left(\left(3.108-8.6 d^{-2 / 3}\right) d\left(k^{2}+s\right)\right) .
$$

Proof. Let us start by showing that $V=V(\boldsymbol{\beta}, \mathbf{r}) \neq 0$ with our choice of $\boldsymbol{\beta}$ and $\mathbf{r}$. From our expression for $V$ in Lemma 6 and our definition of $\boldsymbol{\beta}$, we see that $V=0$ if and only if there exist integers $i, j, k$ and $l$ such that $\alpha_{i}^{p_{k}}=\alpha_{j}^{p_{l}}$. Clearly $i \neq j$, for otherwise $\alpha$ is a root of unity (notice that due to the form of the expression in Lemma 6 along with our choice of $\boldsymbol{\beta}$ and $\mathbf{r}$, if $i=j$ then $k \neq l$ ). Next, by Lemma $5, k=l$ unless $\alpha$ is a root of unity. So we need only consider the case $\alpha_{i}^{p}=\alpha_{j}^{p}$ for some prime $p$.

Define the polynomial $f_{p}(X)$ by

$$
f_{p}(X)=\prod_{j=1}^{d}\left(X-\alpha_{j}^{p}\right) .
$$

By Lemme 7.1.1 of $[1], f_{p}(X) \in \mathbb{Z}[X]$ and is either the minimal polynomial of $\alpha^{p}$ or a power of this minimal polynomial. Now if $\alpha_{i}^{p}=\alpha_{j}^{p}$ for some pair of distinct integers $i$ and $j$, then $f_{p}(X)$ has multiple roots so it 
must be a power of the minimal polynomial of $\alpha^{p}$. But this implies that $\left[\mathbb{Q}\left(\alpha^{p}\right): \mathbb{Q}\right]<[\mathbb{Q}(\alpha): \mathbb{Q}]$, which contradicts one of our hypotheses.

Thus, in what follows, we can suppose that $V$ is non-zero.

With this in mind, let us now obtain a lower bound for $V^{2}$. From the expression in Lemma 6 along with our definitions of $\boldsymbol{\beta}$ and $\mathbf{r}$, we see that

$$
\begin{aligned}
V^{2}= & \left(\prod_{1 \leq i<j \leq d}\left(\alpha_{i}-\alpha_{j}\right)\right)^{2 k^{2}}\left(\prod_{l=1}^{s} \prod_{1 \leq i, j \leq d}\left(\alpha_{i}^{p_{l}}-\alpha_{j}\right)\right)^{2 k} \\
& \times\left(\prod_{l=1}^{s} \prod_{1 \leq i<j \leq d}\left(\alpha_{i}^{p_{l}}-\alpha_{j}^{p_{l}}\right)^{2}\right)\left(\prod_{1 \leq l_{1}<l_{2} \leq s} \prod_{1 \leq i, j \leq d}\left(\alpha_{i}^{p_{l_{1}}}-\alpha_{j}^{p_{l_{2}}}\right)\right)^{2} .
\end{aligned}
$$

Let us denote these products by $A_{1}, A_{2}, A_{3}$ and $A_{4}$ according to their order of appearance. Notice that each of the $A_{i}$ 's, and hence $V^{2}$, is a symmetric function in the $\alpha_{i}$ 's and thus a rational integer since the $\alpha_{i}$ 's are algebraic integers. We shall now determine integers which divide these $A_{i}$ 's.

We first consider $A_{2}$. Let $f(X)$ be the minimal polynomial over $\mathbb{Q}$ of $\alpha$ and notice, by Fermat's little theorem, that

$$
f\left(X^{p}\right) \equiv f(X)^{p} \bmod p
$$

for any prime $p$. Therefore

$$
f\left(\alpha_{i}^{p}\right) \equiv f\left(\alpha_{i}\right)^{p} \equiv 0 \bmod p,
$$

for $1 \leq i \leq d$. Thus $p^{d}$ divides

$$
\prod_{i=1}^{d} f\left(\alpha_{i}^{p}\right)=\prod_{i=1}^{d} \prod_{j=1}^{d}\left(\alpha_{i}^{p}-\alpha_{j}\right)
$$

and therefore $\left(p_{1} \ldots p_{s}\right)^{2 d k}$ divides $A_{2}$. This will provide the main term in our lower bound for $V^{2}$.

Now let us examine $A_{1}$ and $A_{3}$. Notice that $A_{1}$ is $\operatorname{simply} \operatorname{disc}(\alpha)^{k^{2}}$ and the inner product in $A_{3}$ is $\operatorname{disc}\left(\alpha^{p_{l}}\right)$. Since $\alpha$ is an algebraic integer and since we assumed that $\mathbb{Q}(\alpha)=\mathbb{Q}\left(\alpha^{p_{l}}\right)$, these discriminants are both divisible by $D_{\mathbb{Q}(\alpha)}$, the discriminant of $\mathbb{Q}(\alpha)$. Therefore, $D_{\mathbb{Q}(\alpha)}^{k^{2}+s}$ divides $A_{1} A_{3}$.

The reader will notice that the product $A_{4}$ contains a large number of terms in comparison with the other three terms and thus should make a large contribution to a lower bound for $\left|V^{2}\right|$; however, we have been unable to determine a lower bound for $\left|A_{4}\right|$ other than the trivial one: $\left|A_{4}\right| \geq 1$.

Combining these results with our assumption that $V \neq 0$, we find that

$$
\left|V^{2}\right| \geq\left(\prod_{i=1}^{s} p_{i}\right)^{2 d k}\left|D_{\mathbb{Q}(\alpha)}\right|^{k^{2}+s} .
$$

Applying Lemma 4 completes the proof of the lemma. 
We will also need an upper bound. For this, we shall use Hadamard's inequality.

Lemma 8. Suppose that $\boldsymbol{\beta}$ and $\mathbf{r}$ are as above. Then

$$
|V(\boldsymbol{\beta}, \mathbf{r})|^{2} \leq\left(\frac{n^{k^{2}+s}}{\prod_{i=0}^{k-1}(2 i+1)(i !)^{2}}\right)^{d} M(\alpha)^{2 n\left(k+p_{1}+\ldots+p_{s}\right)} .
$$

Proof. By Hadamard's inequality, we have

$$
|V(\boldsymbol{\beta}, \mathbf{r})|^{2} \leq \prod_{j=1}^{m} \prod_{i=0}^{r_{j}-1}\left|v_{i}\left(\beta_{j}\right)\right|^{2}=\left(\prod_{j=1}^{d} \prod_{i=0}^{k-1}\left|v_{i}\left(\beta_{j}\right)\right|^{2}\right)\left(\prod_{j=d+1}^{m}\left|v_{0}\left(\beta_{j}\right)\right|^{2}\right) .
$$

By the definition of $v_{0},\left|v_{0}\left(\beta_{j}\right)\right|^{2} \leq n \max \left(1,\left|\beta_{j}\right|\right)^{2(n-1)}$. Using this along with our definition of $\beta$, we find that

$$
\begin{aligned}
& |V(\boldsymbol{\beta}, \mathbf{r})|^{2} \\
& \leq\left(n^{d s} \prod_{i=1}^{s} \prod_{l=1}^{d}\left(\max \left(1,\left|\alpha_{l}\right|\right)\right)^{2 p_{i}(n-1)}\right)\left(\prod_{j=1}^{d} \prod_{i=0}^{k-1}\left|v_{i}\left(\beta_{j}\right)\right|^{2}\right) \\
& \quad=n^{d s} M(\alpha)^{2(n-1)\left(p_{1}+\ldots+p_{s}\right)}\left(\prod_{j=1}^{d} \prod_{i=0}^{k-1}\left(\sum_{l=i}^{n-1}\left(\begin{array}{l}
l \\
i
\end{array}\right)^{2}\left|\alpha_{j}\right|^{2(l-i)}\right)\right) \\
& \leq n^{d s} M(\alpha)^{2(n-1)\left(p_{1}+\ldots+p_{s}\right)}\left(\prod_{j=1}^{d} \max \left(1,\left|\alpha_{j}\right|\right)^{2(n-1)}\right)\left(\prod_{i=0}^{k-1}\left(\sum_{l=i}^{n-1}\left(\begin{array}{l}
l \\
i
\end{array}\right)^{2}\right)\right)^{d} \\
& \quad=\left(n^{s} \prod_{i=0}^{k-1}\left(\sum_{l=i}^{n-1}\left(\begin{array}{l}
l \\
i
\end{array}\right)^{2}\right)\right)^{d} M(\alpha)^{2(n-1)\left(k+p_{1}+\ldots+p_{s}\right)} .
\end{aligned}
$$

To complete the proof of the lemma, we now use the fact that

$$
\sum_{l=i}^{n-1}\left(\begin{array}{l}
l \\
i
\end{array}\right)^{2} \leq \sum_{l=i}^{n-1} \frac{l^{2 i}}{(i !)^{2}}=\frac{1}{(i !)^{2}} \sum_{l=i}^{n-1} l^{2 i} \leq \frac{1}{(i !)^{2}} \int_{0}^{n} x^{2 i} d x=\frac{n^{2 i+1}}{(2 i+1)(i !)^{2}}
$$

3. Proof of the Theorem. Proceeding by induction, we shall now combine the results we have obtained in the previous section to prove our Theorem.

From the work of Boyd and Smyth, the Theorem holds for $2 \leq d \leq 21$.

Let $\alpha$ be an algebraic number of degree $d \geq 22$ over $\mathbb{Q}$ and assume that the Theorem holds for all $2 \leq d_{1}<d$. Notice that if $\alpha$ is not an algebraic integer then $a_{d} \geq 2$ and $M(\alpha) \geq a_{d} \geq 2$. Therefore we may assume that $\alpha$ is an algebraic integer.

Furthermore, we can also assume that for any prime $p, \mathbb{Q}\left(\alpha^{p}\right)=\mathbb{Q}(\alpha)$. Otherwise, by Lemme 7.1.1 of [1], the polynomial $f_{p}(X)$ defined in the proof 
of Lemma 7 is a power of the minimal polynomial of $\alpha^{p}$. This implies that there exist two distinct integers $i$ and $j$ such that $\alpha_{i}^{p}=\alpha_{j}^{p}$. By Lemme 7.1.2 of [1], there exists a non-zero algebraic integer, $\beta$, which is not a root of unity, of degree less than $d$ with $M(\beta) \leq M(\alpha)$. Since $((\log \log d) /(\log d))^{3}$ is a decreasing function for $d \geq 16$ and since the work of Boyd and Smyth shows that $M(\beta)>(1 / 4)((\log \log 22) /(\log 22))^{3}$ if the degree of $\beta$ over $\mathbb{Q}$ is less than 22 , our inductive hypothesis shows that the Theorem holds.

Let us start by comparing the bounds in Lemmas 7 and 8; taking the logarithm and dividing both sides by $d$, we find that $\log M(\alpha)$ is at least

$$
\begin{aligned}
\left(2 k \theta\left(p_{s}\right)+\right. & \left(k^{2}+s\right)\left(3.108-8.6 d^{-2 / 3}\right)+\sum_{i=0}^{k-1} \log \left((2 i+1)(i !)^{2}\right) \\
& \left.-\left(k^{2}+s\right) \log (d(k+s))\right) /\left(2(k+s)\left(k+p_{1}+\ldots+p_{s}\right)\right) .
\end{aligned}
$$

We will first show that we have

$$
0.56\left(\frac{\log \log d}{\log d}\right)^{3}<\log M(\alpha)
$$

for $d \leq 190$, after which we shall show that

$$
\frac{1}{4}\left(\frac{\log \log d}{\log d}\right)^{3}<\log M(\alpha)
$$

for $d \leq 10000$.

For $k=7$ and $s=11$, the left-hand side of (1) is greater than

$$
\frac{422.1-516 d^{-2 / 3}-60 \log d}{6012} \text {. }
$$

One can compute this quantity for $22 \leq d \leq 94$ to show that it is greater than $0.56((\log \log d) /(\log d))^{3}$. Similarly, one can show that the choice $k=8$ and $s=14$ yields the desired result for $43 \leq d \leq 190$. Finally, letting $k=7$ and $s=17$, we obtain

$$
\frac{1}{4}\left(\frac{\log \log d}{\log d}\right)^{3}<\log M(\alpha),
$$

for $22 \leq d \leq 10000$.

For all $d>10000$, choose

$$
k=k_{1} \frac{\log d}{\log \log d} \quad \text { and } \quad s=s_{1}\left(\frac{\log d}{\log \log d}\right)^{2}
$$

respectively, where the pair $\left(k_{1}, s_{1}\right)$ is contained in some region $\mathcal{A}$ of $\mathbb{R}^{2}$. We shall choose this region so that (1) is at least $((\log \log d) /(\log d))^{3} / 4$ for every pair $\left(k_{1}, s_{1}\right)$ in $\mathcal{A}$ and also so that there is always a pair $\left(k_{1}, s_{1}\right)$ in $\mathcal{A}$ 
such that $k$ and $s$ are positive integers. For now, we shall assume only that $1.2 \leq k_{1} \leq 1.6$ and $1.2 \leq s_{1} \leq 1.51$.

Since $s_{1} \geq 1.2$ and $d>10000$, we have $s>13$, and so we may apply Lemma 2(i) to obtain

$$
2 k \theta\left(p_{s}\right) \geq 2 k_{1} s_{1}\left(\frac{\log d}{\log \log d}\right)^{3}\left(\log s_{1}+2 \log \log d-2 \log \log \log d\right) .
$$

Let us now bound from above the last term in the numerator of (1). Using the expressions for $k$ and $s$ above and the fact that $d>10000$, we have $k+s<2((\log d) /(\log \log d))^{2}$. Thus

$$
\log (d(k+s))<\log d+2 \log \log d+0.7-2 \log \log \log d .
$$

Using Lemma 1 and the above expression for $k$, we have

$$
2 \sum_{i=0}^{k-1} \log (i !)>\left(k_{1} \frac{\log d}{\log \log d}\right)^{2}\left(\log \log d-\log \log \log d+\log k_{1}-1.5\right) .
$$

Since $3.108-8.6 d^{-2 / 3}>3.089$ for $d>10000$, we can bound the last three terms in the numerator of (1) from below by

$$
\begin{array}{r}
-\frac{\left(k_{1}^{2}+s_{1}\right)(\log d)^{3}}{(\log \log d)^{2}}-\left(k_{1}^{2}+2 s_{1}\right) \frac{(\log d)^{2}}{\log \log d}+\left(k_{1}^{2}+2 s_{1}\right) \log \log \log d\left(\frac{\log d}{\log \log d}\right)^{2} \\
+\left(\left(0.89+\log k_{1}\right) k_{1}^{2}+2.39 s_{1}\right)\left(\frac{\log d}{\log \log d}\right)^{2} .
\end{array}
$$

Note that to simplify the argument we have ignored the term $\sum_{i=0}^{k-1} \log (2 i$ +1 ) which is small for large $d$.

Applying Lemma 3 to this last expression, we find that the sum of the last three terms of the numerator of (1) is greater than

$$
-\frac{\left(k_{1}^{2}+s_{1}\right)(\log d)^{3}}{(\log \log d)^{2}}-\frac{2 k_{1} s_{1} \log s_{1}(\log d)^{3}}{(\log \log d)^{2}} .
$$

Combining this expression with our lower bound for the first term of (1), we find that the numerator of (1) is greater than

$$
\frac{\left(4 k_{1} s_{1}-k_{1}^{2}-s_{1}\right)(\log d)^{3}}{(\log \log d)^{2}}-4 k_{1} s_{1} \log \log \log d\left(\frac{\log d}{\log \log d}\right)^{3} .
$$

Since $(\log \log \log d) / \log \log d<0.368$ for $d>10000$, this lower bound yields the still-simpler lower bound

$$
\frac{\left(2.528 k_{1} s_{1}-k_{1}^{2}-s_{1}\right)(\log d)^{3}}{(\log \log d)^{2}} .
$$

Now let us obtain an upper bound for the denominator of (1). Using the expressions for $k$ and $s$ along with the bound in Lemma 2(iii), which is 
possible since we saw above that $s>9$, we find that this denominator is at most

$$
\text { (3) } \begin{aligned}
\frac{2.256 s_{1}^{3}(\log d)^{6}}{(\log \log d)^{5}} & \left(1+\frac{k_{1} \log \log d}{s_{1} \log d}\right) \\
& \times\left(\frac{k_{1}(\log \log d)^{2}}{1.128 s_{1}^{2}(\log d)^{3}}+1+\frac{\log s_{1}-2 \log \log \log d}{2 \log \log d}\right) .
\end{aligned}
$$

We shall show that the product of the last two terms is less than 1 . To demonstrate this we shall prove that the function

$$
\begin{aligned}
g\left(k_{1}, s_{1}, d\right)= & \frac{2 \log \log \log d-\log s_{1}}{2 \log \log d}-\frac{k_{1}(\log \log d)^{2}}{1.128 s_{1}^{2}(\log d)^{3}}-\frac{k_{1} \log \log d}{s_{1} \log d} \\
= & \left(282 s_{1}^{2}(\log d)^{3} \log \log \log d-141 s_{1}^{2}\left(\log s_{1}\right)(\log d)^{3}\right. \\
& -282 k_{1} s_{1}(\log d)^{2}(\log \log d)^{2} \\
& \left.-250 k_{1}(\log \log d)^{3}\right) /\left(282 s_{1}^{2}(\log d)^{3} \log \log d\right)
\end{aligned}
$$

is positive for $d>10000,1.2 \leq s_{1} \leq 1.51$ and $1.2 \leq k_{1} \leq 1.05 s_{1}$. This will imply that the product of the last two terms in (3) can be written as $(1+a)(1-b)$ where $a$ and $b$ are functions of $k_{1}, s_{1}$ and $d$ which satisfy $b>a$, from which our claim follows.

We need only examine the numerator of the expression for $g$. Since $k_{1}$ only occurs in terms being subtracted, we may replace it by $1.05 s_{1}$ in light of our constraint on $k_{1}$. Therefore we need only show that

$2820 s_{1}(\log d)^{3} \log \log \log d-1410 s_{1}\left(\log s_{1}\right)(\log d)^{3}$

$$
-2961 s_{1}(\log d)^{2}(\log \log d)^{2}-2625(\log \log d)^{3}
$$

is positive for $d>10000$ and $1 \leq s_{1} \leq 1.51$, upon dividing by $s_{1}$.

We can rewrite this expression as

$$
\begin{aligned}
& s_{1}(\log d)^{3}\left(2820 \log \log \log d-1410 \log s_{1}-\frac{2961(\log \log d)^{2}}{\log d}\right) \\
& -2625(\log \log d)^{3} \text {. }
\end{aligned}
$$

Now since $(\log \log d)^{2} / \log d<0.54$ for $d>10000$ and since $s_{1} \leq 1.51$, this expression is greater than

$$
69 s_{1}(\log d)^{3}-2625(\log \log d)^{3} .
$$

Since $(\log \log d) / \log d<0.25$ for $d>10000$, this last expression is greater than $41(\log d)^{3}$ for $s_{1} \geq 1.2$ and $d>10000$. Hence $g\left(d, k_{1}, s_{1}\right)$ is positive in the desired region.

Therefore the product in question is less than 1, which implies that

$$
2(k+s)\left(k+p_{1}+\ldots+p_{s}\right) \leq 2.256 s_{1}^{3} \frac{(\log d)^{6}}{(\log \log d)^{5}} .
$$


Combining (2) with the upper bound in (4), we will show that

$$
\frac{2.528 k_{1} s_{1}-k_{1}^{2}-s_{1}}{2.256 s_{1}^{3}}>\frac{1}{4}
$$

for all $1.26 \leq k_{1} \leq 1.51$ and $k_{1}-0.06 \leq s_{1} \leq k_{1}$. Notice that this choice of $k_{1}$ and $s_{1}$ satisfies the conditions set down above. Also, since $d>10000$, there exist $k_{1}$ and $s_{1}$ in this range such that $k$ and $s$ are integers.

Let us now turn to proving this inequality. We shall use ideas from multi-variable calculus. Let

$$
f\left(k_{1}, s_{1}\right)=\frac{2.528 k_{1} s_{1}-k_{1}^{2}-s_{1}}{2.256 s_{1}^{3}} .
$$

Then

$$
\frac{\partial f}{\partial k_{1}}=\frac{158 s_{1}-125 k_{1}}{141 s_{1}^{3}} \text { and } \quad \frac{\partial f}{\partial s_{1}}=\frac{375 k_{1}^{2}-632 k_{1} s_{1}+250 s_{1}}{282 s_{1}^{4}} .
$$

From these expressions, we find that both derivatives are zero only at $k_{1}=125 / 79=1.58 \ldots$ and $s_{1}=15625 / 12482=1.25 \ldots$-but these values do not fall inside our range and hence there are no local minima for such $k_{1}$ and $s_{1}$. Thus we need only look along the boundary to determine the minimum of $f$ for $\left(k_{1}, s_{1}\right)$ in this region. Along the edge formed by the line $k_{1}=1.26$, we have $f\left(1.26, s_{1}\right)=\left(27316 s_{1}-19845\right) /\left(28200 s_{1}^{3}\right)$. For $1.2 \leq s_{1} \leq 1.26$, this function attains its minimum, which is greater than 0.2583 , at $s_{1}=1.26$. Along the edge formed by the line $k_{1}=1.51$, we have $f\left(1.51, s_{1}\right)=\left(140864 s_{1}-114005\right) /\left(112800 s_{1}^{3}\right)$. For $1.45 \leq s_{1} \leq$ 1.51 , the minimum of this function occurs at $s_{1}=1.51$ and is greater than 0.2541. Along the boundary formed by the line $k_{1}=s_{1}+0.06$, $f\left(k_{1}, s_{1}\right)=\left(19100 s_{1}^{2}-12104 s_{1}-45\right) /\left(28200 s_{1}^{3}\right) \geq 0.2624$ - this value occurs for $s=1.45$. Finally, along the boundary formed by the line $k_{1}=s_{1}$, $f\left(k_{1}, s_{1}\right)=\left(191 s_{1}-125\right) /\left(282 s_{1}^{2}\right) \geq 0.2541$ - this value occurs for $s=1.51$.

This completes the proof of the Theorem.

\section{References}

[1] M. J. Bertin et M. Pathiaux-Delefosse, Conjecture de Lehmer et petits nombres de Salem, Queen's Papers in Pure and Appl. Math. 81, Kingston, 1989.

[2] P. E. Blanksby and H. L. Montgomery, Algebraic integers near the unit circle, Acta Arith. 18 (1971), 355-369.

[3] D. W. Boyd, Reciprocal polynomials having small measure, Math. Comp. 35 (1980), 1361-1377.

[4] —, Reciprocal polynomials having small measure. II, ibid. 53 (1989), 355-357.

[5] D. C. Cantor and E. G. Straus, On a conjecture of D. H. Lehmer, Acta Arith. 42 (1982), 97-100; corrigendum, ibid., 327. 
[6] E. Dobrowolski, On a question of Lehmer and the number of irreducible factors of a polynomial, ibid. 34 (1979), 391-401.

[7] A. Dubickas, On a conjecture of A. Schinzel and H. Zassenhaus, ibid. 63 (1993), $15-20$.

[8] M. Laurent, M. Mignotte et Y. Nesterenko, Formes linéaires en deux logarithmes et déterminant d'interpolation, J. Number Theory, to appear.

[9] D. H. Lehmer, Factorization of certain cyclotomic functions, Ann. of Math. (2) 34 (1933), 461-479.

[10] R. Louboutin, Sur la mesure de Mahler d'un nombre algébrique, C. R. Acad. Sci. Paris Sér. I 296 (1983), 707-708.

[11] E. M. Matveev, On the measure of algebraic integers, Mat. Zametki 49 (1991), 152-154 (in Russian).

[12] M. Meyer, Le problème de Lehmer: méthode de Dobrowolski et lemme de Siegel "à la Bombieri-Vaaler", Publ. Math. Univ. P. et M. Curie (Paris VI), 90, Problèmes Diophantiens 1988/89, $\mathrm{N}^{\circ} 5,15 \mathrm{p}$.

[13] G. Poitou, Minorations de discriminants [d'après A.M. Odlyzko], Séminaire Bourbaki 479, Lecture Notes in Math. 567, Springer, 1977, 136-153.

[14] G. Robin, Estimation de la fonction de Tchebychef $\theta$ sur le k-ième nombre premier et grandes valeurs de la fonction $\omega(n)$ nombre de diviseurs premiers de $n$, Acta Arith. 42 (1983), 367-389.

[15] J. B. Rosser and L. Schoenfeld, Approximate formulas for some functions of prime numbers, Illinois J. Math. 6 (1962), 64-94.

[16] A. Schinzel and H. Zassenhaus, A refinement of two theorems of Kronecker, Michigan Math. J. 12 (1965), 81-85.

[17] C. J. Smyth, On the product of the conjugates outside the unit circle of an algebraic integer, Bull. London Math. Soc. 3 (1971), 169-175.

[18] C. L. Stewart, Algebraic integers whose conjugates lie near the unit circle, Bull. Soc. Math. France 106 (1978), 169-176.

DEPARTMENT OF MATHEMATICS

CITY UNIVERSITY

NORTHAMPTON SQUARE

LONDON EC1V 0HB, U.K. 